\title{
Possibility of Poloidal Current Drive by Low Energy Axial NBI into Field-Reversed Configuration Plasma
}

\author{
Takayuki WATANABE, Takahiro URANO and Toshiki TAKAHASHI \\ Department of Electronic Engineering, Gunma University, 1-5-1 Tenjin-Cho, Gunma 376-8515, Japan
}

(Received 19 December 2012 / Accepted 7 February 2013)

\begin{abstract}
Possibility of the poloidal current drive by low energy axial neutral beam injection is studied by a MonteCarlo method for field-reversed configuration (FRC) plasma. In order to suppress the end-loss rate of beam ions, it is found that the separatrix beta of target plasma should be low (presumably less than 0.1). If a beam source of a few $100 \mathrm{eV}$ can be developed, beam ions could be trapped inside the separatrix and poloidal current drive would be possible even for FRC plasma embedded in the open-field region.
\end{abstract}

(c) 2013 The Japan Society of Plasma Science and Nuclear Fusion Research

Keywords: field-reversed configuration, neutral beam injection, poloidal current drive, Störmer region, MonteCarlo simulation

DOI: $10.1585 / \mathrm{pfr} .8 .1203015$

Field-reversed configurations (FRCs) are sustained by the toroidal plasma current and have negligible toroidal field. According to the result of translation experiment [1], a weak but finite toroidal field generated spontaneously in the translation process would stabilize the plasma and prolong the lifetime of FRC. A shear field may also improve confinement of electrons. Therefore, we need to develop an effective technique to introduce the toroidal field. In the present paper, possibility of poloidal current drive of the FRC by a low-energy axial neutral beam injection (NBI) is numerically studied by a Monte-Carlo method.

Axial NBI is believed to be ineffective for FRCs, because significant amount of beam ions are lost as they move along the open field line. In first NBI experiment, the neutral beam (NB) particles of $14 \mathrm{keV}$ are injected obliquely into the FIX-FRC plasma [2], where the beam ions are traveling both inside and outside of the separatrix and confined by the mirror field. Therefore, the beam ions are occasionally subject to the end loss. However, if a low energy beam source would be developed, it might be possible for beam ions to be confined in the separatrix even in the axial NBI case.

Now, we will discuss the equilibrium of which NB particles are ionized deeply inside the separatrix. When the separatrix beta value is high, most of the NB particles are ionized in the edge layer surrounding the separatrix. Consequently, the beam ions tend to move along the open field line, and are lost through the axial end of confinement region. Hence, the target plasma should have low separatrix beta and thin edge layer to confine the beam ions inside the separatrix. We call this desired equilibrium "sharp boundary equilibrium (SBE)". In order to obtain the SBE state, we change the pressure function in the Grad-Shafranov equation. The obtained SBE state in the cylindrical sys-

author'se-mail: t11801656@gunma-u.ac.jp tem $r, z$ is shown in Fig. 1 where the equilibrium with relatively high separatrix beta value (0.82) [3] is also drawn and $\psi_{\mathrm{w}}$ is the poloidal flux function at the chamber wall, whose radius is $r_{\mathrm{w}}$. When the ion and electron temperatures are 90 and $60 \mathrm{eV}$, respectively, the field-null density of SBE is $3.7 \times 10^{21} \mathrm{~m}^{-3}$; the results shown hereinafter are obtained under this condition. As shown in Fig. 1, the separatrix beta value of SBE state is 0.035 , and the width of edge layer is much thinner than ordinary cases. As the den-
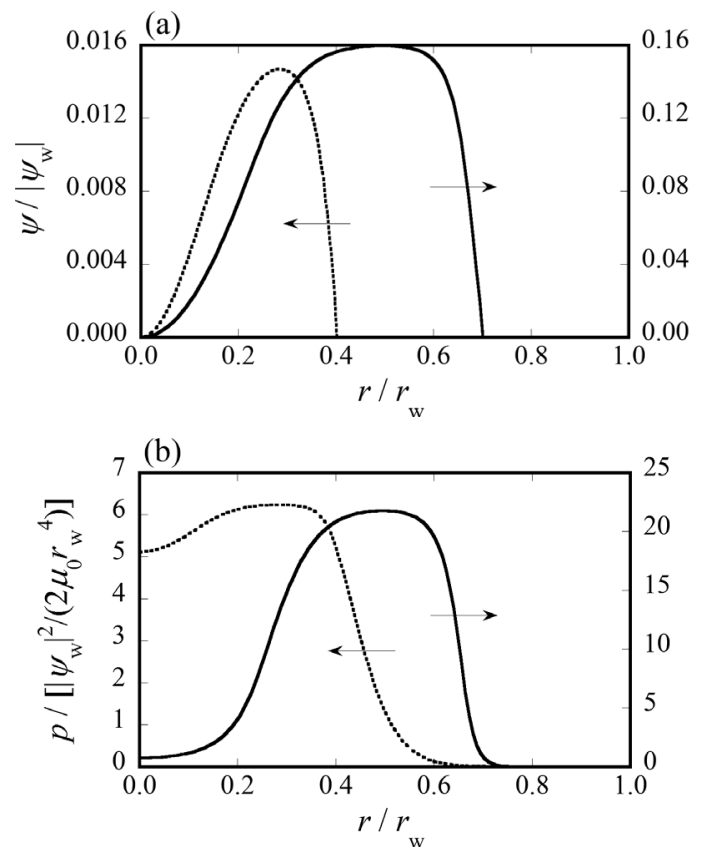

Fig. 1 Radial midplane profile of (a) the poloidal flux and (b) the pressure. The solid lines indicate the sharp boundary equilibrium and the dotted line profile is showing a high separatrix beta value $(0.82)$. 


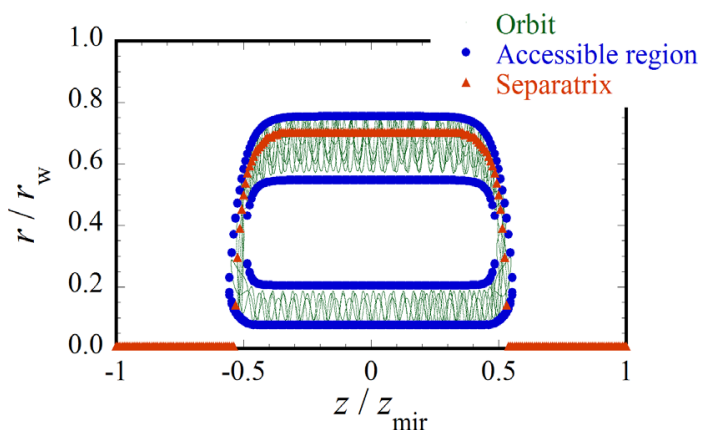

Fig. 2 The beam ion accessibility in $r-z$ plane.

sity in the edge layer is low, less NB particles are ionized outside the separatrix. In order to drive the poloidal current, beam ions can be confined inside the separatrix and drift along the closed field line with small-gyroradius. If the beam energy is high enough to be accessible the open field region, almost all the ions are lost through the end of confinement region. Also since high-energy beam ions exhibit the encircling betatron or figure- 8 orbits $[4,5]$, they never form the poloidal current. On the other hand, smallgyroradius drift particles that are drifting in the toroidal direction by the gradient and curvature of magnetic field can move along the closed magnetic field, and therefore they make up the axial beam current. Three types of beam ion orbit can be classified by calculating the Störmer region [6] within which the beam ions are accessible. The beam ion accessibility in the axisymmetric system is written by

$$
U\left(r, z ; P_{\theta}\right)=\frac{\left(P_{\theta}-q \psi(r, z)\right)^{2}}{2 m r^{2}} \leq H-q \phi(r, z),
$$

where $m$ and $q$ are the mass and charge of the beam ion respectively, $P_{\theta}$ is the canonical angular momentum, $H$ is the Hamiltonian, and $\phi$ is the electrostatic potential that is neglected in the present study.

When the calculated Störmer region for a beam ion is open to the axial end, the ion would be lost rapidly and is not able to drive the poloidal current. If the Störmer region is closed and hollow shape as seen in Fig. 2, the confined beam ion gyrates around the field line near the separatrix and flows in the poloidal direction. As the inequality (1) is dependent only on $H$ and $P_{\theta}$, we can check if the Störmer region is closed and hollow shape like Fig. 2 when the NB particle is ionized. Ionization processes of NB particles by electron impact or charge exchange are reproduced by a Monte-Carlo method [3]. For the equilibrium with high separatrix beta (indicated by the dotted lines in Fig. 1), all NB particles are ionized in the edge layer and outside the separatrix, and are lost without being trapped in the core region. Contrary to this, it is found that most of NB particles are ionized inside the separatrix of the SBE state.

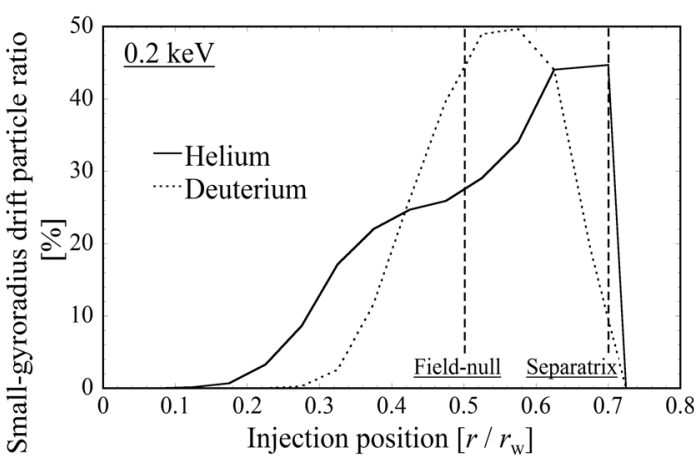

Fig. 3 Radial midplane profile of small-gyroradius drift particle ratio for $0.2-\mathrm{keV}$ beam ions.

Moreover, we find that the beam ion can have a closed and hollow shape Störmer region seen in Fig. 2. For 0.2-keV beam, the ratio of small-gyroradius drift particle is calculated and shown in Fig. 3. The ratio is defined by the particle number of drifting particle versus the total NB particle. Here, axial NBI is done by changing the impact parameter written as the injection position. The ratio for deuterium is peaked near the field-null and steeply decreases toward the separatrix. Since the penetration length and gyroradius for the deuterium case are smaller than helium injection, the Störmer region becomes open shape to the axial end when NB particles are injected toward the separatrix.

We have shown possibility of the poloidal current by low energy axial NBI. Beam ions with the axial momentum are successfully confined in an FRC at the SBE state. The beam current along the field line, however, is shielded by electrons flowing by the drag force from the beam ions. By a use of the Ohkawa's formula [7], one half of the beam current becomes the driven current when the effective charge is 2 [8]. Therefore, the poloidal current drive may be still possible. It generates the toroidal field and localized magnetic shear near the separatrix. Preliminary particle-tracking calculations of electrons show that the generated toroidal field of $0.1 \mathrm{~T}$ suppresses the electron end loss rate. Detailed discussion on shear field generation and stability and confinement improvement by low energy axial NBI will be presented in a subsequent paper.

[1] H.Y. Guo et al., Phys. Rev. Lett. 95, 175001 (2005).

[2] T. Asai et al., Phys. Plasmas 7, 2294 (2000).

[3] T. Takahashi et al., Phys. Plasmas 11, 3131 (2004).

[4] J.M. Finn and R.N. Sudan, Nucl. Fusion 22, 1443 (1982).

[5] Y. Hayakawa et al., Nucl. Fusion 42, 1075 (2002).

[6] C. Störmer, The polar aurora (Oxford University Press, UK, 1955).

[7] T. Ohkawa, Nucl. Fusion 10, 185 (1970).

[8] J.T. Slough and A.L. Hoffman, Phys. Fluids B 2, 797 (1990). 